OPEN ACCESS

Edited by:

Bruce M. Damon,

Vanderbilt University Medical Center

United States

Reviewed by:

Fabio Esposito,

Università degli Studi di Milano, Italy

Crystal L. Coolbaugh,

Vanderbilt University, United States

${ }^{*}$ Correspondence:

Adamantios Arampatzis a.arampatzis@hu-berlin.de

Specialty section:

This article was submitted to

Exercise Physiology,

a section of the journal

Frontiers in Physiology

Received: 29 March 2017 Accepted: 30 May 2017

Published: 16 June 2017

Citation:

Mersmann F, Charcharis G, Bohm S and Arampatzis A (2017) Muscle and Tendon Adaptation in Adolescence: Elite Volleyball Athletes Compared to

Untrained Boys and Girls.

Front. Physiol. 8:417.

doi: 10.3389/fphys.2017.00417

\section{Muscle and Tendon Adaptation in Adolescence: Elite Volleyball Athletes Compared to Untrained Boys and Girls}

\author{
Falk Mersmann ${ }^{1,2}$, Georgios Charcharis ${ }^{1,2}$, Sebastian Bohm ${ }^{1,2}$ and \\ Adamantios Arampatzis ${ }^{1,2 *}$ \\ ${ }^{1}$ Department of Training and Movement Sciences, Humboldt-Universität zu Berlin, Berlin, Germany, ${ }^{2}$ Berlin School of \\ Movement Science, Berlin, Germany
}

Though the plasticity of human tendons is well explored in adults, it is still unknown how superimposed mechanical loading by means of athletic training affects the properties of tendons during maturation. Due to the increased responsiveness of muscle to mechanical loading, adolescence is an important phase to investigate the effects of training on the mechanical properties of tendons. Hence, in the present study we compared vastus lateralis (VL) architecture, muscle strength of the knee extensor muscles and patellar tendon mechanical properties of male and female adolescent elite athletes to untrained boys and girls. Twenty-one adolescent volleyball athletes ( $A$; 16.7 \pm 1 years; 12 boys, 9 girls) and 24 similar-aged controls (C; $16.7 \pm 1$ years; 12 boys and girls, respectively) performed maximum isometric contractions on a dynamometer for the assessment of muscle strength and, by integrating ultrasound imaging, patellar tendon mechanical properties. Respective joint moments were calculated using an inverse dynamics approach and an electromyography-based estimation of antagonistic contribution. Additionally, the VL pennation angle, fascicle length and muscle-thickness were determined in the inactive state by means of ultrasound. Adolescent athletes produced significantly greater knee extension moments (normalized to body mass) compared to controls (A: $4.23 \pm 0.80 \mathrm{Nm} / \mathrm{kg}$, C: $3.57 \pm 0.67 \mathrm{Nm} / \mathrm{kg} ; p=0.004$ ), and showed greater $V L$ thickness and pennation angle $(+38 \%$ and $+27 \% ; p<0.001)$. Tendon stiffness (normalized to rest length) was also significantly higher in athletes ( $A$ : $86.0 \pm 27.1 \mathrm{kN} /$ strain, C: $70.2 \pm 18.8 \mathrm{kN} / \mathrm{strain} ; p=0.04$ ), yet less pronounced compared to tendon force (A: $5785 \pm 1146 \mathrm{~N}, \mathrm{C}: 4335 \pm 1015 \mathrm{~N} ; p<0.001$ ), which resulted in higher levels of tendon strain during maximum contractions in athletes ( $A$ : 8.0 $\pm 1.9 \%, \mathrm{C}: 6.4 \pm 1.8 \% ; p=0.008)$. We conclude that athletic volleyball training provides a more efficient stimulus for muscle compared to tendon adaptation, which results in an increased demand placed upon the tendon by the working muscle in adolescent volleyball athletes. Besides implications for sport performance, these findings might have important consequences for the risk of tendon overuse injury.

Keywords: muscle, tendon, adaptation, athletes, adolescence, tendinopathy, imbalance 


\section{INTRODUCTION}

Tendons transmit the forces generated by the muscle to the skeleton and, thus, feature a crucial role in the production of torques around joints for movement. The viscoelastic properties of tendons importantly contribute to the force- and powergenerating capacity of the muscle-tendon unit by optimizing the operating range with regard to the force-velocity and force-length relationship and storage and release of mechanical strain energy (Hof et al., 1983; Ettema et al., 1990; Roberts, 1997; Kawakami and Fukunaga, 2006). Thus, there is a clear relationship between the properties of tendons and human movement performance, for example for running (Arampatzis et al., 2006; Fletcher et al., 2010; Albracht and Arampatzis, 2013), sprinting (Stafilidis and Arampatzis, 2007; Kubo et al., 2011b), jumping (Bojsen-Møller et al., 2005), rapid force production (Waugh et al., 2013) or balance recovery performance (Karamanidis et al., 2008).

On the other hand, the tissue deformation during loading makes the tendon susceptible for injury. Cyclic (or constant) high-magnitude strains applied to a tendon can cause fatigue damage and even rupture (Woo, 1982; Wren et al., 2003; Lavagnino et al., 2006; Legerlotz et al., 2013; Veres et al., 2013). Therefore, tendons are able to adapt to changes in their mechanical environment and, for example, respond to an increase in muscle force potential with a modulation of its mechanical properties. The increase of tendon stiffness in response to biologically effective repetitive mechanical stimulation over a certain time is mediated by changes of the material properties (Kubo et al., 2001; Arampatzis et al., 2007, 2010; Malliaras et al., 2013; Bohm et al., 2014) and, following long-term loading, radial tendon growth (Rosager et al., 2002; Magnusson and Kjaer, 2003; Kongsgaard et al., 2005; Couppé et al., 2013).

The plasticity of tendons is well explored in human adults (Bohm et al., 2015; Wiesinger et al., 2015). In contrast, there is basically no information on the adaptation of human tendons in response to mechanical loading during childhood and adolescence. Waugh et al. (2014) were the first to investigate the effects of mechanical loading on Achilles tendon adaptation in pre-pubertal children in a longitudinal study and found a significant increase of muscle strength and a concomitant increase of tendon stiffness following a resistance exercise intervention. However, puberty is associated with profound changes of the musculoskeletal and endocrine system, which affect the plasticity of muscle and likely tendon as well. Estrogen and particularly testosterone increase the anabolic responsiveness of muscle to mechanical loading (Vingren et al., 2010; Hansen and Kjaer, 2014) and a recent meta-analysis provided evidence for an increase of muscle strength plasticity during and after peak height velocity (Moran et al., 2016). Youth athletes feature markedly greater muscle size compared to untrained adolescents, as demonstrated by Kanehisa et al. (1995b, 2003) as well as Hoshikawa et al. (2011), and, though it has not been demonstrated thus far experimentally, it is likely that changes in muscle architecture also contribute to training-induced gains in strength in adolescents (Aagaard et al., 2001). How the change in the hormonal environment affects tendon adaptation is on the other hand virtually unknown and, thus, the findings of Waugh et al. (2014) on pre-pubertal children are not necessarily representative for pubertal children or adolescents. Second, the mechanical stimulus provided by Waugh and colleagues in their machine-based resistance training intervention (i.e., high magnitude loading, long contraction durations) is known to facilitate tendon mechanical properties (Bohm et al., 2015), yet the dominant type of loading for young athletes is sport-specific and might not automatically provide an efficient stimulus for both muscle and tendon adaptation.

As the incidence of tendon overuse injuries seems to increase during adolescence (Stracciolini et al., 2014; Simpson et al., 2016) and pose a major threat to athletes from jump disciplines (Lian et al., 2005), it is crucial (a) to deepen our understanding of the adaptive processes of muscle and tendon in adolescents and (b) to detect potential factors that could promote tendon overuse pathology. Therefore, the present study investigated the properties of the knee extensor muscle-tendon unit of male and female adolescent elite volleyball athletes compared to untrained boys and girls. With regard to the seemingly lower responsiveness of the tendon to plyometric loading compared to muscle (Kubo et al., 2007; Sáez-Sáez de Villarreal et al., 2010; Bohm et al., 2014) and our recent findings of a deficient modulation of tendon stiffness in relation to muscle strength development in adolescent athletes (Mersmann et al., 2016), we hypothesized to find markedly greater muscle strength in volleyball athletes compared with untrained adolescents, mediated by greater muscle thickness and fascicle pennation angles but only moderately higher tendon stiffness. The resultant higher mechanical demand (i.e., strain) placed upon the tendon during maximum effort muscle contractions could have important implications for the risk of tendon overload injury in adolescent volleyball athletes.

\section{MATERIALS AND METHODS}

\section{Participants and Experimental Design}

Twenty-four recreationally active adolescents [12 males, 12 females; $\leq 4 \mathrm{~h}$ of training per week, including school sports over the last 12 month (average values were $2.6 \pm 0.9 \mathrm{~h}$ ); henceforth referred to as controls] and 21 similar-aged elite volleyball athletes (12 male and 9 female athletes of the junior national team; $\geq 16$ $\mathrm{h}$ of sport-specific training per week) participated in the present study. At the time of data acquisition the athletes had participated in national elite training for $9 \pm 5$ month, which comprised $\sim 3 \mathrm{~h}$ of strength training, $\sim 4 \mathrm{~h}$ of athletic training (i.e., jump and sprint drills, and core stability training) and $\geq 9 \mathrm{~h}$ of ball practice per week. As the effects of oral contraceptives (OC) on in vivo patellar tendon properties are most likely negligible (Hansen et al., 2013), we decided to include girls using OC in the present study (Athletes: $n=1 / 9$; controls: $n=2 / 12$; none of which were long-term OC users, i.e., $<1$ year of use).

The study was carried out in accordance with the recommendations of the university ethics committee with written informed consent from all subjects. All subjects (and the respective legal guardians when necessary) gave written informed consent in accordance with the Declaration of Helsinki. The 
protocol was approved by the university ethics committee. The measurements of muscle strength (i.e., knee extension moments), vastus lateralis architecture and patellar tendon mechanical properties were carried out on the dominant leg (i.e., leading leg in the spike jump in athletes or leg used for kicking a ball in controls) following a standardized warm-up including $5 \mathrm{~min}$ of ergometer cycling, 10 submaximal jumps and 10 submaximal isometric knee extension contractions as accustoming and preconditioning.

\section{Measurement of Maximum Knee Joint Moment}

For the assessment of knee extensor muscle strength, the participants performed three maximum voluntary isometric knee extension contractions (MVC) on a dynamometer (Biodex Medical System 3, Shirley, NY, USA) at resting knee joint angles of $65^{\circ}, 70^{\circ}$, and $75^{\circ}$ (neutral full knee extension $=0^{\circ}$, values refer to the joint angle determined via the dynamometer). The resting angles were chosen based on our experience that participants reach their approximate optimum angle during contractions from these starting positions. The trunk angle was set to $85^{\circ}$ (neutral full hip extension $=0^{\circ}$ ) and the hip as fixed to the dynamometer seat using a non-elastic strap (Figure 1). Kinematic data were recorded using a Vicon motion capture system (version 1.7.1; Vicon Motion Systems, Oxford, UK) integrating eight cameras operating at $250 \mathrm{~Hz}$. Five reflective markers were fixed to the following anatomical landmarks: greater trochanter, lateral, and medial femoral epicondyles, and malleoli. The electromyographic (EMG) activity of the lateral head of the biceps femoris was recorded using two bipolar surface electrodes (Blue Sensor N, Ambu GmbH, Bad Nauheim, Germany) fixed over the mid-portion of the muscle belly with an inter-electrode distance of $2 \mathrm{~cm}$ after shaving and cleaning the skin. EMG data was captured at 1,000 Hz (Myon m320RX; Myon, Baar, Switzerland) and transmitted to the Vicon system via a 16-channel A-D converter.

Due to the non-rigidity of the human-dynamometer system (Arampatzis et al., 2004), the knee joint angle that was reached in the trails where the maximum knee extension moments were generated was $54 \pm 4^{\circ}$, calculated in post-processing from the kinematic model. The resultant knee joint moments were calculated based on the inverse dynamics approach proposed by Arampatzis et al. (2004), which takes into account the inevitable axes misalignments of the knee joint and dynamometer during the course of the contraction as well as moments due to gravity. To account for the latter, an additional trial was recorded, where a passive knee extension was driven by the dynamometer at $5 \%$ s with the shank of the participants fixed to the dynamometer lever pad. The contribution of the antagonistic muscles during the isometric contractions was estimated in the calculation of the maximum knee extension moments using the approach described by Mademli et al. (2004). In short, an EMG-activity knee flexion moment relationship was established on the basis of two additional knee flexion trials featuring an EMG-activity that was slightly lower and higher compared to the activity registered during the maximum knee extension trials, respectively.

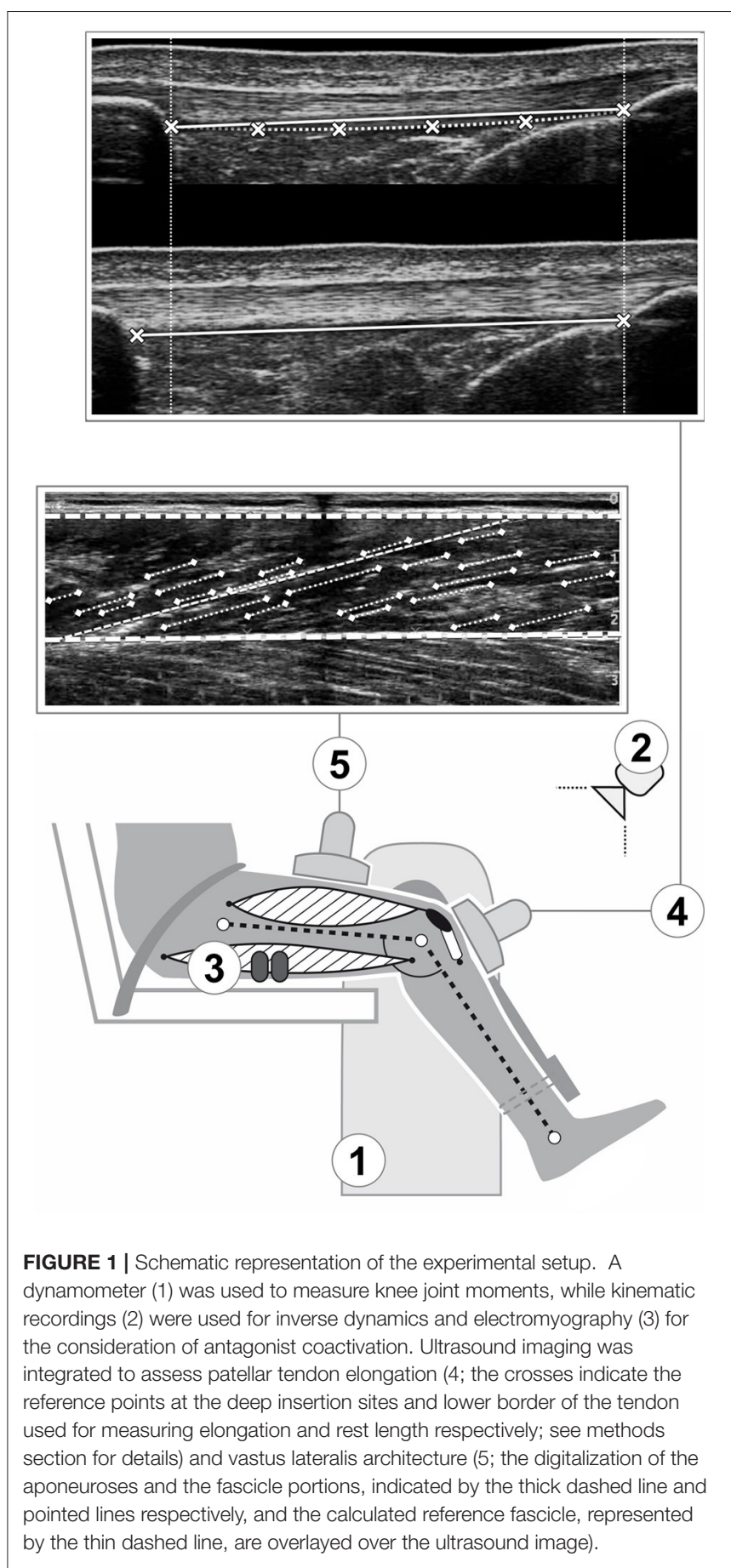

\section{Measurement of Vastus Lateralis Muscle Architecture}

Vastus lateralis architecture was assessed at a knee joint angle of $60^{\circ}$, which has been reported earlier to be the approximate optimum knee angle for force production of the knee extensors (Herzog et al., 1990). A 10-cm linear ultrasound probe $(7.5 \mathrm{MHz}$; My Lab60; Esaote, Genova, Italy; probe: linear array (LA923), depth: $7.4 \mathrm{~cm}$, focal point: 0.9 and 1.9, no image filter) was 
placed over the belly of the inactive muscle in its longitudinal axis at $\sim 60 \%$ thigh length (average location of the maximum anatomical cross-sectional area; Mersmann et al., 2015). The ultrasound images were analyzed offline using a custom written MATLAB interface (version R2012a; MathWorks, Natick, MA, USA). The upper and deeper aponeuroses were defined by setting reference points along the aponeuroses that were approximated by a linear least squares fitting. Subsequently, we digitized the visible features of multiple fascicles (on average $22 \pm 5$ ) and calculated a reference fascicle based on the average inclination of the fascicle portions and the distance of the aponeuroses (Figure 1; Mersmann et al., 2014). The pennation angle refers to the angle between the reference fascicle and the deeper aponeurosis and fascicle length is reported normalized to femur length (average value of two measurements from the greater trochanter to the lateral epicondyle, identified by palpation, by means of a measuring tape).

\section{Measurement of Patellar Tendon Mechanical Properties}

To establish the force-elongation behavior of the patellar tendon, the ultrasound probe was fixed overlying the patellar tendon in the sagittal plane using a modified knee brace (i.e., similar probe and settings as described above). The elongation of the tendon was captured at $25 \mathrm{~Hz}$ during five trials of isometric ramp contractions (i.e., steadily increasing effort from rest to maximum in $\sim 5 \mathrm{~s}$ ). The resting knee joint angle for the ramp contractions was chosen for each participant according to the MVC trial in which the highest moments were achieved.

The knee extension moments were calculated using the same considerations as described above for the MVC measurement (i.e., experimental setup, inverse dynamics approach, and correction for antagonistic contribution; see 2.2). The tendon force was then calculated by dividing the knee extension moment by the tendon moment arm. In 19 athletes it was possible to assess the moment arm directly from magnetic resonance images as described earlier (Mersmann et al., 2014). In all other participants, the moment arms were predicted based on sex, body height and mass, using the regression equation reported by Mersmann et al. (2016). The moment arms were adjusted to the respective knee joint angle position using the data of Herzog and Read (1993).

The ultrasound images were synchronized offline with the data recorded with the Vicon system using an externally induced voltage peak, which could be identified in both the ultrasound images and the analog data stream. Patellar tendon elongation during the contractions (Figure 1) was determined manually by tracking the deep insertion of the tendon at the patellar apex and the tibial tuberosity frame-by-frame using a custom-written MATLAB interface. To account for tendon slackness at rest, elongation was measured when the distance between the deep insertion points exceeded tendon rest length, which in turn was measured using a spline fit through the deep insertion marks and four additional points along the lower border of the slack tendon. The tracking was done by two experienced observers (F.M. and G.C.) and the force-elongation relationship of the five trials of each participant was averaged, using the highest common force value as peak force. This approach provides excellent reliability $(\geq 0.95)$ and observer-independence (Schulze et al., 2012). The resultant force-elongation curve was fitted using a second-order polynomial, and tendon stiffness was calculated between 50 and $100 \%$ of the peak tendon force. As the length of a tendon has significant effects on its stiffness (Butler et al., 1978), we accounted for the anthropometric differences between the groups by also calculating normalized tendon stiffness (i.e., the product of stiffness and rest length). In one participant (male, control group), it was not possible to analyze patellar tendon elongation due to ultrasound image artifacts during the contraction.

\section{Statistics}

The statistical analysis was conducted in SPSS (version 20.0; IBM, Armonk, NY, USA). We performed a two-way analysis of variances with the fixed factors training (i.e., controls, athletes) and sex (i.e., male, female). Normality of the standardized residuals was tested using the KolmogorovSmirnov test with Lilliefors correction (Lilliefors, 1967) and Levene's test was applied to test homoscedasticity. If normality or homoscedasticity was violated, we separately tested differences between athletes and controls, and males and females respectively, using the Mann-Whitney-Wilcoxon test and Bonferroni adjustment (adjusted $p$-values, denominated $p_{\text {adj }}$, will be reported). The alpha level for all tests was set to 0.05 . The effect size $f$ for significant observations were calculated in G*Power (Version 3.1.6; HHU, Düsseldorf, Germany; Faul et al., 2007), based on either the partial eta squared or the group means and pooled standard deviation (for non-parametrically tested parameters). The subscript Training or Sex indicates if the effect size refers to differences between controls and athletes or males and females, respectively. Effect sizes of $0.1 \leq f<0.25$ will be referred to as small, $0.25 \leq f<0.5$ as medium and $f \geq 0.5$ as large (Cohen, 2013).

\section{RESULTS}

There was no significant difference of age between the four groups $(p=0.9)$, but athletes compared to controls as well as males compared to females had significantly greater body height $\left(f_{\text {Training }}=1.91, p<0.001 ; f_{\mathrm{Sex}}=1.27, p<0.001\right)$, mass $\left(f_{\text {Training }}\right.$ $\left.=0.50, p_{\text {adj }}=0.004 ; f_{\text {Sex }}=0.46, p_{\text {adj }}=0.008\right)$ and femur lengths $\left(f_{\text {Training }}=1.60, p<0.001 ; f_{\text {Sex }}=0.56, p=0.001\right.$; Table 1, respectively). There were no significant training-by-sex interactions on the anthropometric parameters $(p>0.05)$.

Absolute $\left(f_{\text {Training }}=1.07, p<0.001 ; f_{\text {Sex }}=1.11, p<0.001\right)$ and normalized knee extensor muscle strength $\left(f_{\text {Training }}=0.48\right.$, $\left.p=0.004 ; f_{\text {Sex }}=0.51, p=0.002\right)$, and absolute $\left(f_{\text {Training }}=1.18\right.$, $\left.p<0.001 ; f_{\text {Sex }}=1.09, p<0.001\right)$ and normalized resultant knee joint moments $\left(f_{\text {Training }}=0.56, p=0.001 ; f_{\text {Sex }}=0.45, p=0.006\right)$ were significantly greater in athletes and males, respectively (Table 2), without significant training-by-sex interactions $(p>$ $0.05)$. Further, there was a tendency toward lower coactivation in athletes $\left(f_{\text {Training }}=0.32, p_{\text {adj }}=0.056\right)$, yet no significant differences between males and females $\left(p_{\mathrm{adj}}=1.0\right.$; Table 2$)$. 
TABLE 1 | Antrhopometric data of the adolescent controls and volleyball athletes.

\begin{tabular}{|c|c|c|c|c|}
\hline & \multicolumn{2}{|c|}{ Controls } & \multicolumn{2}{|c|}{ Athletes } \\
\hline & $\begin{array}{c}\text { Male } \\
(n=12)\end{array}$ & $\begin{array}{l}\text { Female } \\
(n=12)\end{array}$ & $\begin{array}{c}\text { Male } \\
(n=12)\end{array}$ & $\begin{array}{c}\text { Female } \\
(n=9)\end{array}$ \\
\hline Age [years] & $16.8 \pm 1.1$ & $16.6 \pm 0.9$ & $16.8 \pm 1.0$ & $16.7 \pm c$ \\
\hline Height $[\mathrm{cm}]^{\star \#}$ & $175.0 \pm 4.4$ & $162.1 \pm 8.1$ & $195.2 \pm 4.0$ & $181.6 \pm 3$ \\
\hline Mass $[\mathrm{kg}]^{\star \#}$ & $67.9 \pm 11.5$ & $61.9 \pm 17.7$ & $86.1 \pm 7.2$ & $68.0 \pm 5$ \\
\hline Femur length $[\mathrm{cm}]^{\star \#}$ & $41.5 \pm 2.3$ & $38.1 \pm 3.6$ & $48.4 \pm 1.5$ & $46.4 \pm 1$ \\
\hline
\end{tabular}

Values are means \pm standard deviation. ${ }^{*}$ Significant difference between athletes and controls, " significant difference between males and females, $p<0.05$.

We found significantly greater vastus lateralis muscle thickness in athletes compared to controls $\left(f_{\text {Training }}=1.12, p\right.$ $<0.001)$ as well as in males compared to females $\left(f_{\mathrm{Sex}}=0.37\right.$, $p=0.023)$, without significant training-by-sex interactions $(p$ $=0.29$; Figure 2A). Further, athletes featured greater pennation angles compared to controls $\left(f_{\text {Training }}=0.75, p<0.001\right.$ ), while there were no significant differences between males and females $(p=0.72)$ or training-by-sex interactions $(p=0.3$; Figure $2 \mathbf{B})$. No significant effects or interactions were found on normalized fascicle length ( $p>0.05$; Figure 2C).

In athletes compared to controls, we found greater maximum patellar tendon force $\left(f_{\text {Training }}=0.82, p<0.001\right.$; Figure 3A), normalized tendon stiffness $\left(f_{\text {Training }}=0.34, p=0.04\right.$; Figure 3B), tendon strain $\left(f_{\text {Training }}=0.42, p_{\text {adj }}=0.016\right.$; Figure 3C) and elongation during maximum contractions $\left(f_{\text {Training }}=0.55, p=0.001\right.$; Table 3$)$, tendon moment arm $\left(f_{\text {Training }}=1.58, p<0.001\right.$; Table 3$)$, and rest length $\left(f_{\text {Training }}=\right.$ $0.57, p=0.001$; Table 3), but no significant difference in absolute tendon stiffness $(p=0.26$; Table 3$)$. Tendon force $\left(f_{\text {Sex }}=0.83\right.$, $p<0.001)$, moment arm $\left(f_{\text {Sex }}=1.55, p<0.001\right)$ and rest length $\left(f_{\text {Sex }}=0.48, p=0.004\right)$ were higher in males compared to females. However, there were no significant differences between sexes with regard to absolute $(p=0.57)$ or normalized tendon stiffness $(p=0.17)$, strain $(p=0.49)$ and elongation $(p=0.12)$ or training-by-sex interactions in general $(p>0.05)$.

\section{DISCUSSION}

The present study investigated the effects of mechanical loading in terms of athletic volleyball training on the properties of the knee extensor muscle-tendon unit in male and female adolescents. The results demonstrate that, irrespective of sex and anthropometric differences, both muscle and tendon show clear differences between trained and untrained boys and girls. We found a significantly greater muscle strength capacity in the athletes compared to the control group and corresponding differences in vastus lateralis muscle thickness and pennation angle. Similarly, athletes demonstrated greater normalized patellar tendon stiffness, indicating that the tendon adapts to mechanical loading before full maturation of the musculoskeletal system. However, the only medium effects of training status on normalized tendon stiffness compared to the large effects on tendon force ( $f=0.34$ and 0.84 , respectively) suggest an imbalance in the adaptation of muscle and tendon, subjecting the
TABLE 2 | Knee joint moments and antagonistic coactivation of adolescent controls and volleyball athletes.

\begin{tabular}{|c|c|c|c|c|}
\hline & \multicolumn{2}{|c|}{ Controls } & \multicolumn{2}{|c|}{ Athletes } \\
\hline & $\begin{array}{c}\text { Male } \\
(n=12)\end{array}$ & $\begin{array}{l}\text { Female } \\
(n=12)\end{array}$ & $\begin{array}{c}\text { Male } \\
(n=12)\end{array}$ & $\begin{array}{c}\text { Female } \\
(n=9)\end{array}$ \\
\hline MVC [Nm] $]^{\star} \#$ & $264.2 \pm 57.2$ & $192.8 \pm 25.5$ & $385.6 \pm 48.3$ & $260.6 \pm 48.0$ \\
\hline $\begin{array}{l}\text { Normalized MVC } \\
{[\mathrm{Nm} / \mathrm{kg}]^{\star} \#}\end{array}$ & $3.89 \pm 0.52$ & $3.25 \pm 0.67$ & $4.52 \pm 0.74$ & $3.86 \pm 0.74$ \\
\hline $\begin{array}{l}\text { Resultant moment } \\
{[\mathrm{Nm}]^{\star} \#}\end{array}$ & $237.9 \pm 54.2$ & $174.7 \pm 22.2$ & $355.2 \pm 38.7$ & $245.2 \pm 45.2$ \\
\hline $\begin{array}{l}\text { Normalized } \\
\text { resultant moment } \\
{[\mathrm{Nm} / \mathrm{kg}]^{*} \#}\end{array}$ & $3.50 \pm 0.54$ & $2.96 \pm 0.66$ & $4.15 \pm 0.58$ & $3.63 \pm 0.68$ \\
\hline $\begin{array}{l}\text { Antagonistic } \\
\text { coactivation [\%] }\end{array}$ & $13.0 \pm 7.9$ & $11.3 \pm 6.4$ & $9.1 \pm 6.0$ & $7.0 \pm 3.4$ \\
\hline
\end{tabular}

Values are means \pm standard deviation. Moments were normalized to body mass. Antagonistic coactivation is the antagonistic moment normalized to the resultant knee joint moment. MVC, Maximum voluntary contraction. *Significant difference between athletes and controls, "Significant difference between males and females, $p<0.05$; $\left({ }^{*}\right)$ tendency toward a difference between controls and athletes, $p_{\text {adj }}=0.056$.

tendons of athletes to higher levels of strain during maximum contractions compared to controls. Therefore, our hypotheses were confirmed.

It is well established that physical training increases muscle strength even in childhood (Falk and Eliakim, 2003; Matos and Winsley, 2007; Legerlotz et al., 2016) and, thus, our findings of greater knee extensor strength in trained compared to untrained adolescents were not surprising. Similarly, our findings of greater vastus lateralis thickness, pennation angle, and, in tendency, reduced antagonistic coactivation in athletes are in line with earlier reports of muscle morphological (Fukunaga et al., 1992; Kanehisa et al., 1995a, 2003; Daly et al., 2004; Mersmann et al., 2016) and neuronal adaptations (Ramsay et al., 1990; Ozmun et al., 1994) as potential contributors to training-induced increases of strength in young athletes. However, this is, to our knowledge, the first study to report differences in patellar tendon mechanical properties between an athletic and untrained adolescent population. Waugh et al. (2014) investigated the effects of a strength training intervention on the mechanical properties of the Achilles tendon in pre-pubertal children and reported a significant increase of tendon stiffness. More recently, a cross-sectional comparison of tendon thickness between 500 adolescent athletes from different sports provided an indication of tendon plasticity in response to increased loading for the patellar tendon as well (Cassel et al., 2016). Earlier work of our group already suggested that the patellar tendon is responsive to mechanical stimulation, but it was not possible to differentiate the effects of mechanical loading and maturation (Mersmann et al., 2017) or to directly compare the mechanical properties of the patellar tendon between athletes and controls due to inhomogeneous sample composition with regard to sex (Mersmann et al., 2016). The different normalized patellar tendon stiffness between athletes and controls in the present study provide evidence that also the patellar tendon adapts to mechanical loading before adulthood. With regard to 


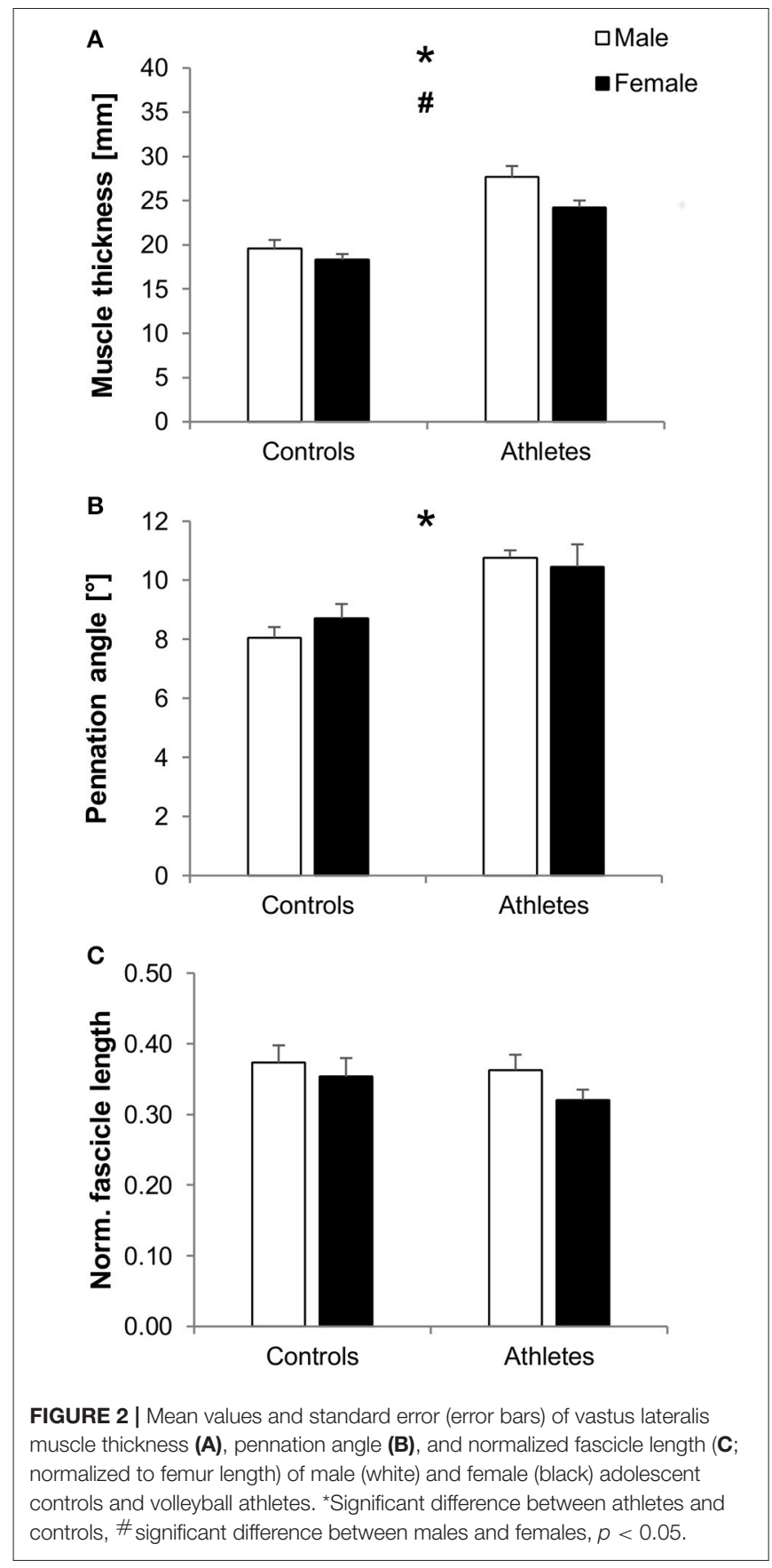

the marked effect of athletic training on muscle strength, the increase of tendon stiffness serves two important mechanical functions. First, it improves the performance capacity of the muscle-tendon unit. Higher tendon stiffness is associated with a reduction of electromechanical delay (Waugh et al., 2014), increased rate of torque development (Bojsen-Møller et al., 2005; Waugh et al., 2013) and jump performance (BojsenMøller et al., 2005; Burgess et al., 2007), and it maintains muscle fascicle kinetics within an optimal operating range when the force production during a movement task (e.g., jumping) increases due to training (Lichtwark and Wilson,

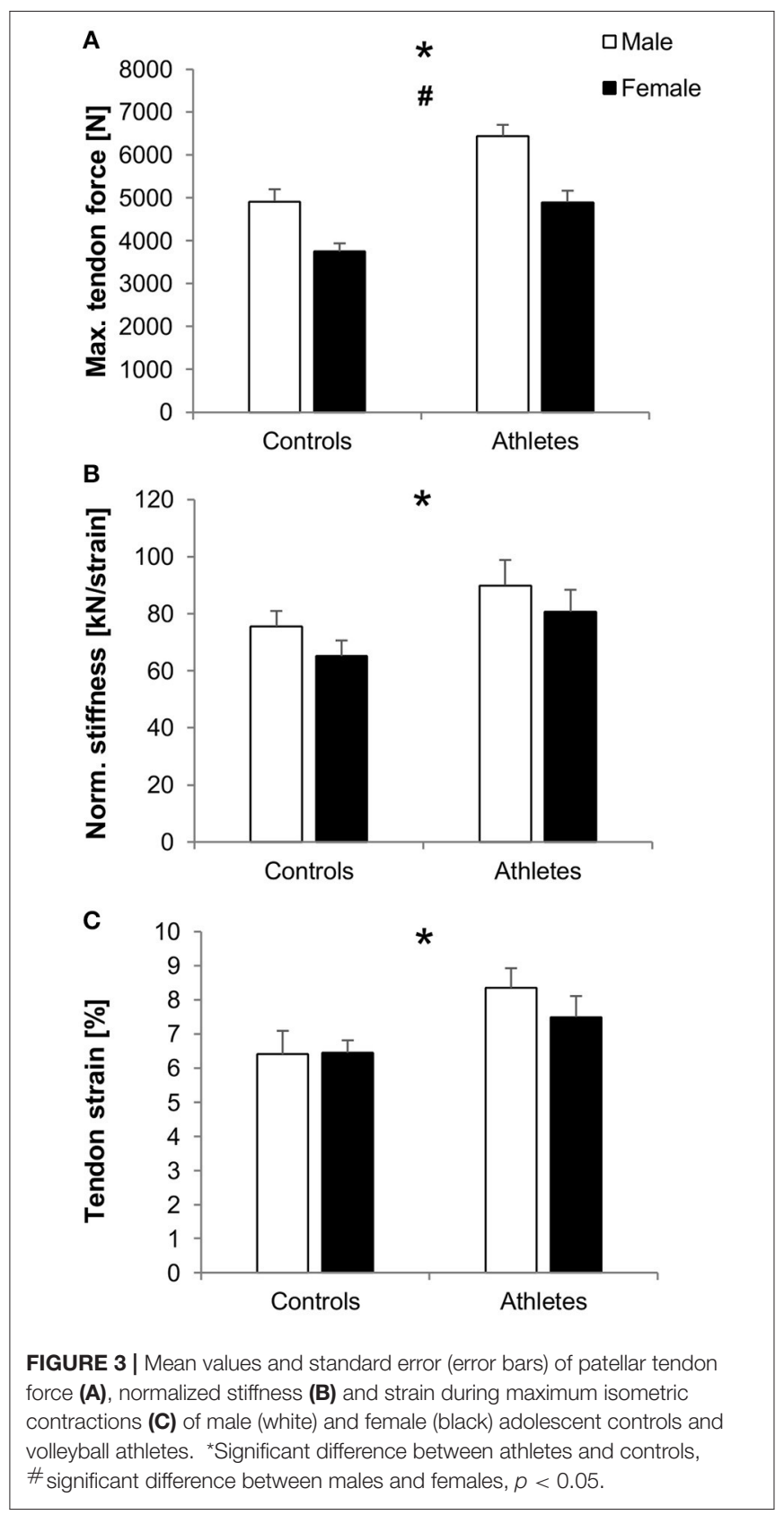

TABLE 3 | Patellar tendon properties of adolescent controls and volleyball athletes.

\begin{tabular}{lccccc}
\hline & \multicolumn{2}{c}{ Controls } & & \multicolumn{2}{c}{ Athletes } \\
\cline { 2 - 3 } \cline { 5 - 6 } \cline { 5 - 6 } & $\begin{array}{c}\text { Male } \\
(\boldsymbol{n}=\mathbf{1 1})\end{array}$ & $\begin{array}{c}\text { Female } \\
(\boldsymbol{n}=\mathbf{1 2})\end{array}$ & & $\begin{array}{c}\text { Male } \\
(\boldsymbol{n}=\mathbf{1 2})\end{array}$ & $\begin{array}{c}\text { Female } \\
(\boldsymbol{n}=\mathbf{9})\end{array}$ \\
\hline Moment arm [mm]*\# & $53.6 \pm 1.3$ & $49.2 \pm 2.0$ & & $59.8 \pm 3.2$ & $53.5 \pm 3.0$ \\
Rest length [mm]*\# & $51.6 \pm 4.5$ & $46.6 \pm 4.5$ & & $56.0 \pm 5.8$ & $52.5 \pm 3.4$ \\
Stiffness [N/mm] & $1474 \pm 375$ & $1392 \pm 352$ & & $1650 \pm 670$ & $1560 \pm 518$ \\
$\begin{array}{l}\text { Maximum elongation } \\
{[\mathrm{mm}]^{*}}\end{array}$ & $3.30 \pm 1.26$ & $2.98 \pm 0.64$ & & $4.71 \pm 1.37$ & $3.94 \pm 1.05$ \\
& & & & &
\end{tabular}

Values are means \pm standard deviation. *Significant difference between athletes and controls, "Significant difference between males and females, $p<0.05$. 
2007). Second, the modulation of stiffness may serve as a protective mechanism against unphysiological levels of strain induced by the increased force-generating capacity of the trained muscle, since the ultimate strain of tendons is considered to be relatively constant (Abrahams, 1967; Loitz et al., 1989; LaCroix et al., 2013; Shepherd and Screen, 2013). However, besides increased normalized stiffness, the athletes in the present study featured increased levels of tendon strain during maximum muscle contractions as well, which is in accordance with earlier findings of our group (Mersmann et al., 2016) and indicates an imbalanced adaptation of muscle and tendon. Tendon strain during maximum contractions is an indicator of how the integrity of the tissue is challenged by the working muscle. Wren et al. (2003) demonstrated that the initial strain induced in the tendon by a given load predicts the lifetime of the tendinous tissue during static and cyclic loading. The increased demand placed upon the tendon by the working muscle we observed in athletes could increase the risk of accumulating microdamage and predispose for tendon injury (Butler et al., 1978; Fung et al., 2009; Legerlotz et al., 2013), especially in athletes that are subjected to high frequencies of maximum jumping in their sportive activity (Bahr and Bahr, 2014). The high prevalence of tendinopathy in volleyball athletes (Lian et al., 2005) and in vivo reports of increased levels of tendon strain in patients with tendinopathy (Arya and Kulig, 2010; Child et al., 2010) further support the idea that an imbalanced adaptation of muscle and tendon in athletes might increase the risk to develop tendinopathy, though certainly more direct evidence is needed to support this assumption.

The higher normalized stiffness compared to untrained adolescents as well as the imbalance between muscle strength and tendon stiffness are most likely related to the type of mechanical loading the athletes are subjected to. Systematic investigations on the specific effects of different modes of mechanical stimulation on human tendons in vivo demonstrated that high magnitude loading (in terms of tendon force and corresponding strain) effectively promote tendon stiffness (Arampatzis et al., 2007, 2010; Kongsgaard et al., 2007; Malliaras et al., 2013). Highlevel strain application has been associated with greater tendon cell deformation (Arnoczky et al., 2002) and collagen fiber recruitment (Kastelic et al., 1980; Hansen et al., 2002), which are considered important factors for the transmission of extracellular matrix strains into cellular responses (Lavagnino et al., 2008). Direct measurements (Finni et al., 2000) and estimations of patellar tendon forces (Janssen et al., 2013) suggest tendon loads to exceed five times body weight during jumping and landing, which makes it reasonable to assume that athletic volleyball training provides sufficient loading in terms of load magnitude to induce tendon adaptation. High tendon strain rates during plyometric loading and the associated fluid-flow dependent shear stress on tendon cells might additionally stimulate tendon metabolism (Haut and Haut, 1997; Archambault et al., 2002; Lavagnino et al., 2008). Interestingly though, most longitudinal intervention-studies that applied plyometric exercise (over 8-14 weeks) failed to elicit significant adaptive changes of human tendons (Kubo et al., 2007; Fouré et al., 2009, 2010; Houghton et al., 2013), even at high loading magnitudes (Bohm et al., 2014). In consequence, an increase of muscle strength without an adequate modulation of stiffness induced by plyometric loading can result in increased levels of tendon deformation (Kubo et al., 2007), which is commonly not observed following high-intensity loading with long contraction durations (e.g., Kongsgaard et al., 2007; Arampatzis et al., 2007, 2010; Malliaras et al., 2013). It has been argued that short strain durations of plyometric regimen or high-frequency loadrelaxation cycles might compromise the effectiveness of the mechanotransduction at the tendon level (Arampatzis et al., 2010; Bohm et al., 2014). Therefore, it seems well possible that only long-term habitual plyometric loading results in a notable modulation of tendon stiffness, but that the effects are still less pronounced compared to the associated and more clearly established increases of muscle strength (Sáez-Sáez de Villarreal et al., 2010). With regard to the greater body mass of the athletes in our study, it cannot be excluded, of course, that increased habitual loading during everyday activities partly contributed to the greater tendon stiffness compared to the control group. However, body mass was not correlated to normalized tendon stiffness in the present study $(r=0.013, p=0.94)$ and data by Waugh et al. (2011) sugests that, at least in the Achilles tendon, a clear association between body mass and tendon stiffness only exists during pre-pubertal growth and not in adulthood. Thus, it is reasonable to conclude that the sport-specific loading was the main determinant for both the greater normalized tendon stiffness as well as the imbalance between muscle strength and tendon mechanical properties.

An alternative or additional explanation for the imbalance between muscle strength and tendon stiffness, indicated by the increased levels of tendon strain during maximum contractions in athletes compared to controls, could be differences in the plasticity of muscle and tendon as a function of maturation. Earlier work of our group already provided evidence that under the influence of maturation and superimposed mechanical loading the development of muscle morphology and function might precede adaptive and developmental processes at the tendon level in adolescent volleyball athletes (Mersmann et al., $2014,2017)$. The results of a recent meta-analysis indicate an increase in the responsiveness of the neuromuscular system to mechanical loading early in adolescence (Moran et al., 2016), which is likely in part related to the muscle-anabolic effects of sex hormones (Vingren et al., 2010; Hansen and Kjaer, 2014). The effects of the rapid increase of circulating sex hormones on tendon plasticity during growth on the other hand are basically unknown (Hansen and Kjaer, 2014). Evidence on human adults suggest that estrogens reduce collagen synthesis in response to exercise, indicating a reduced adaptability of the tendinous system in women compared to men (Hansen and Kjaer, 2014; for reviews see Magnusson et al., 2007). In the present study, however, the differences between athletic and untrained adolescents of both patellar tendon mechanical properties and tendon strain (as an indicator of musculotendious imbalances) were similar in boys and girls (i.e., no significant training-by-sex interaction: $p=0.94$ and 0.44 , respectively). Therefore, it is well possible that sex-related differences in the responsiveness of tendinous tissue to mechanical loading unfold with the formation of the tendon core tissue at the end of adolescence (Heinemeier et al., 2013) and long-term exposure 
to elevated levels of circulating estrogens (Bryant et al., 2008; Hansen and Kjaer, 2014). However, due to a lack of comparable data on adult volleyball athletes with a similar training history compared to the adolescents of the present study, the additional influence of maturation on the balance of musculotendinous adaptations to loading still remains an assumption. Clinical evidence shows that the probability of non-contact soft-tissue injury in adults rises when training loads are increased rapidly (Gabbett, 2016) and differences between muscle and tendon in the responsiveness to distinct mechanical stimuli (Arampatzis et al., 2007, 2010; Kubo et al., 2007) as well as in the time course of adaptation (Kubo et al., 2010, 2011a) are issues that affect the balance of muscle and tendon adaptation in adult athletes as well. Nevertheless, it is evident that there is a need to increase our understanding of the complex interaction of mechanical loading and changes of the hormonal milieu on tendon plasticity in general, and with regard to adolescence in particular.

The World Health Organization defines adolescence as a period ranging roughly from 10 to 19 years of age (World health organization: department of child adolescent health development, 2001), yet a distinction between early adolescence (i.e., 10-14 years) and late adolescence (i.e., 15-19 years) has become common more recently (Sawyer et al., 2012) to partly account for the drastic physical, cognitive and socio-emotional development during the overall period of adolescence. Since it is barely possible to adequately control for biological age with non-invasive measures (Malina et al., 2015), this study investigated late adolescent cohorts following the assumption that the differences in biological and chronological age are most pronounced in early adolescence. Therefore, it is unclear if the observations of the present study are representative for other stages of development. Considering the observations of increased tendon stress during the longitudinal growth of the muscletendon unit in early adolescence (Neugebauer and Hawkins, 2012), this could be important maturational phase to address with future experimental designs.

Due to the cross-sectional design of the study, the time course of muscle and tendon adaptation in relation to training history (i.e., $6 \pm 3$ years of volleyball practice, $9 \pm 5$ month of elite-level training) and/or maturation also remains unclear. The fluctuations of both muscle and tendon properties during a competitive season, which was observed in a recent study of our group (Mersmann et al., 2016), suggests that also the time point of data acquisition could be important for a comparison to untrained individuals. In the present study, it was not possible to adjust the scheduling of the measurements to a specific time point in the competitive season of the athletes. However, since the fluctuations over time reported earlier (e.g., MVC: 5\%, stiffness: $8 \%$, strain: $14 \%$; Mersmann et al., 2016) were markedly lower as the differences observed between the athletes and controls of the present study (MVC: 45\%, normalized stiffness: 23\%, strain: 24\%) it is unlikely that this limitation affected our main findings and conclusions.

The selection of elite volleyball athletes as trained group was based on the sport-specific loading profile (i.e., high intensity plyometric loading) and the urgent need for a better understanding of muscle and tendon adaptation due to the high incidence of tendinopathy in that group (Lian et al., 2005). The generalizability of our findings to other athletic populations is speculative. It seems well possible that differentially graded adaptations of muscle and tendon might occur in other sports that incorporate types of loading that lead to rapid strength gains (i.e., high intensity muscle contractions; Seynnes et al., 2007; DeFreitas et al., 2011) or more effectively stimulate muscle compared to tendon adaptation (i.e., moderate intensity loading, plyometric loading; Arampatzis et al., 2007, 2010; Kubo et al., 2007; Bohm et al., 2014). Our findings might therefore be relevant for the design of training programs in sports such as Basketball, Soccer or athletic jumping disciplines as well. However, this assumption warrants verification in future studies.

In conclusion, the present study provides evidence that, irrespective of sex, adolescent volleyball athletes feature markedly greater muscle strength, mediated by greater muscle thickness and pennation angle as well as reduced antagonistic coactivation, and greater normalized tendon stiffness compared to untrained adolescents. However, increased levels of tendon strain during maximum contractions in athletes indicate an imbalance in the development of muscle strength and tendon stiffness that might be partly due to (a) suboptimal tendon mechanical stimulation by sport-specific loading and (b) deviations in the temporal dynamics of muscle and tendon adaptation during adolescence. The potential contribution of musculotendinous imbalances to the increasing risk of tendon overload injury during adolescence (Stracciolini et al., 2014; Simpson et al., 2016) highlight the importance to further increase our understanding of muscle and tendon plasticity during growth and maturation as well as to evaluate the potential of implementing loading regimen that effectively facilitate tendon mechanical properties (Bohm et al., 2015) into the athletic training of adolescents for injury prevention.

\section{AUTHOR CONTRIBUTIONS}

FM and AA conceived the experiment; FM, GC, and SB performed the experiments; all authors substantially contributed to data analysis; FM and AA interpreted the data; FM drafted the manuscript and GC, SB, and AA made important intellectual contributions during revision. All authors approved the final version of the manuscript and agree to be accountable for the content of the work.

\section{FUNDING}

This study is part of the research project "Resistance Training in Youth Athletes (http://www.uni-potsdam.de/kraftprojekt/ english.php) that was funded by the German Federal Institute of Sport Science (ZMVI1-08190114-18).

\section{ACKNOWLEDGMENTS}

We thank our colleagues Arno Schroll, Robert Marzilger, and Jacob Alekian for their support during data acquisition and analysis. 


\section{REFERENCES}

Aagaard, P., Andersen, J. L., Dyhre-Poulsen, P., Leffers, A. M., Wagner, A., Magnusson, S. P., et al. (2001). A mechanism for increased contractile strength of human pennate muscle in response to strength training: changes in muscle architecture. J. Physiol. (Lond) 534, 613-623. doi: 10.1111/j.1469-7793.2001.t01-1-00613.x

Abrahams, M. (1967). Mechanical behaviour of tendon in vitro. A preliminary report. Med. Biol. Eng. 5, 433-443.

Albracht, K., and Arampatzis, A. (2013). Exercise-induced changes in triceps surae tendon stiffness and muscle strength affect running economy in humans. Eur. J. Appl. Physiol. 113, 1605-1615. doi: 10.1007/s00421-012-2585-4

Arampatzis, A., De Monte, G., Karamanidis, K., Morey-Klapsing, G., Stafilidis, S., and Brüggemann, G.-P. (2006). Influence of the muscle-tendon unit's mechanical and morphological properties on running economy. J. Exp. Biol. 209, 3345-3357. doi: 10.1242/jeb.02340

Arampatzis, A., Karamanidis, K., and Albracht, K. (2007). Adaptational responses of the human Achilles tendon by modulation of the applied cyclic strain magnitude. J. Exp. Biol. 210, 2743-2753. doi: 10.1242/jeb.003814

Arampatzis, A., Karamanidis, K., De Monte, G., Stafilidis, S., MoreyKlapsing, G., and Bruggemann, G. (2004). Differences between measured and resultant joint moments during voluntary and artificially elicited isometric knee extension contractions. Clin. Biomech. 19, 277-283. doi: 10.1016/j.clinbiomech.2003.11.011

Arampatzis, A., Peper, A., Bierbaum, S., and Albracht, K. (2010). Plasticity of human Achilles tendon mechanical and morphological properties in response to cyclic strain. J. Biomech. 43, 3073-3079. doi: 10.1016/j.jbiomech.2010. 08.014

Archambault, J. M., Elfervig-Wall, M. K., Tsuzaki, M., Herzog, W., and Banes, A. J. (2002). Rabbit tendon cells produce MMP-3 in response to fluid flow without significant calcium transients. J. Biomech. 35, 303-309. doi: 10.1016/S0021-9290(01)00217-2

Arnoczky, S. P., Lavagnino, M., Whallon, J. H., and Hoonjan, A. (2002). In situ cell nucleus deformation in tendons under tensile load; a morphological analysis using confocal laser microscopy. J. Orthop. Res. 20, 29-35. doi: 10.1016/S0736-0266(01)00080-8

Arya, S., and Kulig, K. (2010). Tendinopathy alters mechanical and material properties of the Achilles tendon. J. Appl. Physiol. 108, 670-675. doi: 10.1152/japplphysiol.00259.2009

Bahr, M. A., and Bahr, R. (2014). Jump frequency may contribute to risk of jumper's knee: a study of interindividual and sex differences in a total of 11,943 jumps video recorded during training and matches in young elite volleyball players. Br. J. Sports Med. 48, 1322-1326. doi: 10.1136/bjsports-2014-093593

Bohm, S., Mersmann, F., and Arampatzis, A. (2015). Human tendon adaptation in response to mechanical loading: a systematic review and meta-analysis of exercise intervention studies on healthy adults. Sports Med. Open 1:7. doi: 10.1186/s40798-015-0009-9

Bohm, S., Mersmann, F., Tettke, M., Kraft, M., and Arampatzis, A. (2014). Human Achilles tendon plasticity in response to cyclic strain: effect of rate and duration. J. Exp. Biol. 217, 4010-4017. doi: 10.1242/jeb.112268

Bojsen-Møller, J., Magnusson, S. P., Rasmussen, L. R., Kjaer, M., and Aagaard, P. (2005). Muscle performance during maximal isometric and dynamic contractions is influenced by the stiffness of the tendinous structures. J. Appl. Physiol. 99, 986-994. doi: 10.1152/japplphysiol.01305.2004

Bryant, A. L., Clark, R. A., Bartold, S., Murphy, A., Bennell, K. L., Hohmann, E., et al. (2008). Effects of estrogen on the mechanical behavior of the human Achilles tendon in vivo. J. Appl. Physiol. 105, 1035-1043. doi: 10.1152/japplphysiol.01281.2007

Burgess, K. E., Connick, M. J., Graham-Smith, P., and Pearson, S. J. (2007). Plyometric vs. isometric training influences on tendon properties and muscle output. J. Strength Cond. Res. 21, 986-985. doi: 10.1519/R-20235.1

Butler, D. L., Grood, E. S., Noyes, F. R., and Zernicke, R. F. (1978). Biomechanics of ligaments and tendons. Exerc. Sport Sci. Rev. 6, 125-181.

Cassel, M., Carlsohn, A., Fröhlich, K., John, M., Riegels, N., and Mayer, F. (2016). Tendon adaptation to sport-specific loading in adolescent athletes. Int. J. Sports Med. 37, 159-164. doi: 10.1055/s-0035-1559772

Child, S., Bryant, A. L., Clark, R. A., and Crossley, K. M. (2010). Mechanical properties of the achilles tendon aponeurosis are altered in athletes with achilles tendinopathy. Am. J. Sports Med. 38, 1885-1893. doi: 10.1177/0363546510366234

Cohen, J. (2013). Statistical Power Analysis for the Behavioral Sciences. London: Routledge.

Couppé, C., Kongsgaard, M., Aagaard, P., Vinther, A., Boesen, M., Kjaer, M., et al. (2013). Differences in tendon properties in elite badminton players with or without patellar tendinopathy. Scand. J. Med. Sci. Spor. 23, e89-95. doi: $10.1111 /$ sms.12023

Daly, R. M., Saxon, L., Turner, C. H., Robling, A. G., and Bass, S. L. (2004). The relationship between muscle size and bone geometry during growth and in response to exercise. Bone 34, 281-287. doi: 10.1016/j.bone.2003.11.009

DeFreitas, J. M., Beck, T. W., Stock, M. S., Dillon, M. A., and Kasishke, P. R. (2011). An examination of the time course of training-induced skeletal muscle hypertrophy. Eur. J. Appl. Physiol. 111, 2785-2790. doi: 10.1007/s00421-011-1905-4

Ettema, G. J., van Soest, A. J., and Huijing, P. A. (1990). The role of series elastic structures in prestretch-induced work enhancement during isotonic and isokinetic contractions. J. Exp. Biol. 154, 121-136.

Falk, B., and Eliakim, A. (2003). Resistance training, skeletal muscle and growth. Pediatr. Endocrinol. Rev. 1, 120-127.

Faul, F., Erdfelder, E., Lang, A.-G., and Buchner, A. (2007). G*Power 3: a flexible statistical power analysis program for the social, behavioral, and biomedical sciences. Behav. Res. Methods 39, 175-191. doi: 10.3758/BF03193146

Finni, T., Komi, P., and Lepola, V. (2000). In vivo human triceps surae and quadriceps femoris muscle function in a squat jump and counter movement jump. Eur. J. Appl. Physiol. 83, 416-426. doi: 10.1007/s004210000289

Fletcher, J. R., Esau, S. P., and MacIntosh, B. R. (2010). Changes in tendon stiffness and running economy in highly trained distance runners. Eur. J. Appl. Physiol. 110, 1037-1046. doi: 10.1007/s00421-010-1582-8

Fouré, A., Nordez, A., and Cornu, C. (2010). Effects of plyometric training on plantar flexor mechanical properties. Comput. Methods Biomech. Biomed. Engin. 13, 57-59. doi: 10.1080/10255842.2010.493723

Fouré, A., Nordez, A., Guette, M., and Cornu, C. (2009). Effects of plyometric training on passive stiffness of gastrocnemii and the musculo-articular complex of the ankle joint. Scand. J. Med. Sci. Spor. 19, 811-818. doi: 10.1111/j.1600-0838.2008.00853.x

Fukunaga, T., Funato, K., and Ikegawa, S. (1992). The effects of resistance training on muscle area and strength in prepubescent age. Ann. Physiol. Anthropol. 11, 357-364.

Fung, D. T., Wang, V. M., Laudier, D. M., Shine, J. H., Basta-Pljakic, J., Jepsen, K. J., et al. (2009). Subrupture tendon fatigue damage. J. Orthop. Res. 27, 264-273. doi: $10.1002 /$ jor.20722

Gabbett, T. J. (2016). The training-injury prevention paradox: should athletes be training smarter andharder? Br. J. Sports Med. 50, 273-280. doi: 10.1136/bjsports-2015-095788

Hansen, K. A., Weiss, J. A., and Barton, J. K. (2002). Recruitment of tendon crimp with applied tensile strain. J. Biomech. Eng. 124, 72-77. doi: 10.1115/1.1427698

Hansen, M., Couppe, C., Hansen, C. S. E., Skovgaard, D., Kovanen, V., Larsen, J. O., et al. (2013). Impact of oral contraceptive use and menstrual phases on patellar tendon morphology, biochemical composition, and biomechanical properties in female athletes. J. Appl. Physiol. 114, 998-1008. doi: 10.1152/japplphysiol.01255.2012

Hansen, M., and Kjaer, M. (2014). Influence of sex and estrogen on musculotendinous protein turnover at rest and after exercise. Exerc. Sport Sci. Rev. 42, 183-192. doi: 10.1249/JES.0000000000000026

Haut, T. L., and Haut, R. C. (1997). The state of tissue hydration determines the strain-rate-sensitive stiffness of human patellar tendon. J. Biomech. 30, 79-81.

Heinemeier, K. M., Schjerling, P., Heinemeier, J., Magnusson, S. P., and Kjaer, M. (2013). Lack of tissue renewal in human adult Achilles tendon is revealed by nuclear bomb (14)C. FASEB J. 27, 2074-2079. doi: 10.1096/fj.12-225599

Herzog, W., Abrahamse, S. K., and Keurs ter, H. E. (1990). Theoretical determination of force-length relations of intact human skeletal muscles using the cross-bridge model. Pflug. Arch. 416, 113-119.

Herzog, W., and Read, L. J. (1993). Lines of action and moment arms of the major force-carrying structures crossing the human knee joint. J. Anat. 182 (Pt 2), 213-230.

Hof, A. L., Geelen, B. A., and Van den Berg, J. (1983). Calf muscle moment, work and efficiency in level walking; role of series elasticity. J. Biomech. 16, 523-537. 
Hoshikawa, Y., Muramatsu, M., Iida, T., Ii, N., Nakajima, Y., and Kanehisa, H. (2011). Sex differences in the cross-sectional areas of psoas major and thigh muscles in high school track and field athletes and nonathletes. J. Physiol. Anthropol. 30, 47-53. doi: 10.1080/03014468100005351

Houghton, L. A., Dawson, B. T., and Rubenson, J. (2013). Effects of plyometric training on achilles tendon properties and shuttle running during a simulated cricket batting innings. J. Strength Cond. Res. 27, 1036-1046. doi: 10.1519/JSC.0b013e3182651e7a

Janssen, I., Steele, J. R., Munro, B. J., and Brown, N. A. T. (2013). Predicting the patellar tendon force generated when landing from a jump. Med. Sci. Sport Exer. 45, 927-934. doi: 10.1249/MSS.0b013e31827f0314

Kanehisa, H., Funato, K., Kuno, S., Fukunaga, T., and Katsuta, S. (2003), Growth trend of the quadriceps femoris muscle in junior Olympic weight lifters: an 18-month follow-up survey. Eur. J. Appl. Physiol. 89, 238-242. doi: 10.1007/s00421-003-0802-x

Kanehisa, H., Ikegawa, S., Tsunoda, N., and Fukunaga, T. (1995a). Strength and cross-sectional areas of reciprocal muscle groups in the upper arm and thigh during adolescence. Int. J. Sports Med. 16, 54-60. doi: 10.1055/s-2007972964

Kanehisa, H., Yata, H., Ikegawa, S., and Fukunaga, T. (1995b). A cross-sectional study of the size and strength of the lower leg muscles during growth. Eur. J. Appl. Physiol. O. 72, 150-156.

Karamanidis, K., Arampatzis, A., and Mademli, L. (2008). Age-related deficit in dynamic stability control after forward falls is affected by muscle strength and tendon stiffness. J. Electromyogr. Kines 18, 980-989. doi: 10.1016/j.jelekin.2007.04.003

Kastelic, J., Palley, I., and Baer, E. (1980). A structural mechanical model for tendon crimping. J. Biomech. 13, 887-893. doi: 10.1016/0021-9290(80)90177-3

Kawakami, Y., and Fukunaga, T. (2006). New insights into in vivo human skeletal muscle function. Exerc. Sport Sci. Rev. 34, 16-21. doi: 10.1097/00003677-200601000-00005

Kongsgaard, M., Aagaard, P., Kjaer, M., and Magnusson, S. P. (2005). Structural Achilles tendon properties in athletes subjected to different exercise modes and in Achilles tendon rupture patients. J. Appl. Physiol. 99, 1965-1971. doi: 10.1152/japplphysiol.00384.2005

Kongsgaard, M., Reitelseder, S., Pedersen, T. G., Holm, L., Aagaard, P., Kjaer, M., et al. (2007). Region specific patellar tendon hypertrophy in humans following resistance training. Acta Physiol. (Oxf). 191, 111-121. doi: 10.1111/j.1748-1716.2007.01714.x

Kubo, K., Ikebukuro, T., Maki, A., Yata, H., and Tsunoda, N. (2011a). Time course of changes in the human Achilles tendon properties and metabolism during training and detraining in vivo. Eur. J. Appl. Physiol. 112, 2679-2691. doi: $10.1007 / \mathrm{s} 00421-011-2248-\mathrm{x}$

Kubo, K., Ikebukuro, T., Yata, H., Tomita, M., and Okada, M. (2011b). Morphological and mechanical properties of muscle and tendon in highly trained sprinters. J. Appl. Biomech. 27, 336-344. doi: 10.1123/jab.27.4.336

Kubo, K., Ikebukuro, T., Yata, H., Tsunoda, N., and Kanehisa, H. (2010). Time course of changes in muscle and tendon properties during strength training and detraining. J. Strength Cond. Res. 24, 322-331. doi: 10.1519/JSC.0b013e3181c865e2

Kubo, K., Kanehisa, H., Ito, M., and Fukunaga, T. (2001). Effects of isometric training on the elasticity of human tendon structures in vivo. J. Appl. Physiol. 91, 26-32.

Kubo, K., Morimoto, M., Komuro, T., Yata, H., Tsunoda, N., Kanehisa, H., et al. (2007). Effects of plyometric and weight training on muscle-tendon complex and jump performance. Med. Sci. Sport Exer. 39, 1801-1810. doi: $10.1249 / \mathrm{mss} .0 \mathrm{~b} 013 \mathrm{e} 31813 \mathrm{e} 630 \mathrm{a}$

LaCroix, A. S., Duenwald-Kuehl, S. E., Lakes, R. S., and Vanderby, R. (2013). Relationship between tendon stiffness and failure: a metaanalysis. J. Appl. Physiol. 115, 43-51. doi: 10.1152/japplphysiol.01449.2012

Lavagnino, M., Arnoczky, S. P., Egerbacher, M., Gardner, K. L., and Burns, M. E. (2006). Isolated fibrillar damage in tendons stimulates local collagenase mRNA expression and protein synthesis. J. Biomech. 39, 2355-2362. doi: 10.1016/j.jbiomech.2005.08.008

Lavagnino, M., Arnoczky, S. P., Kepich, E., Caballero, O., and Haut, R. C. (2008). A finite element model predicts the mechanotransduction response of tendon cells to cyclic tensile loading. Biomech. Model. Mechanobiol. 7, 405-416. doi: $10.1007 / \mathrm{s} 10237-007-0104-\mathrm{z}$
Legerlotz, K., Jones, G. C., Screen, H. R. C., and Riley, G. P. (2013). Cyclic loading of tendon fascicles using a novel fatigue loading system increases interleukin-6 expression by tenocytes. Scand. J. Med. Sci. Spor. 23, 31-37. doi: 10.1111/j.1600-0838.2011.01410.x

Legerlotz, K., Marzilger, R., Bohm, S., and Arampatzis, A. (2016). Physiological adaptations following resistance training in youth athletes-A narrative review. Pediatr. Exerc. Sci. 28, 501-520. doi: 10.1123/pes.2016-0023

Lian, O. B., Engebretsen, L., and Bahr, R. (2005). Prevalence of jumper's knee among elite athletes from different sports: a cross-sectional study. Am. J. Sports Med. 33, 561-567. doi: 10.1177/0363546504270454

Lichtwark, G. A., and Wilson, A. M. (2007). Is Achilles tendon compliance optimised for maximum muscle efficiency during locomotion? J. Biomech. 40, 1768-1775. doi: 10.1016/j.jbiomech.2006.07.025

Lilliefors, H. W. (1967). On the Kolmogorov-Smirnov test for normality with mean and variance unknown. JASA 62, 399-402. doi: $10.1080 / 01621459.1967 .10482916$

Loitz, B. J., Zernicke, R. F., Vailas, A. C., Kody, M. H., and Meals, R. A. (1989). Effects of short-term immobilization versus continuous passive motion on the biomechanical and biochemical properties of the rabbit tendon. Clin. Orthop. Relat. Res. 244, 265-271.

Mademli, L., Arampatzis, A., Morey-Klapsing, G., and Bruggemann, G. (2004). Effect of ankle joint position and electrode placement on the estimation of the antagonistic moment during maximal plantarflexion. J. Electromyogr. Kines 14, 591-597. doi: 10.1016/j.jelekin.2004.03.006

Magnusson, S. P., Hansen, M., Langberg, H., Miller, B., Haraldsson, B., Westh, E. K., et al. (2007). The adaptability of tendon to loading differs in men and women. Int. J. Exp. Pathol. 88, 237-240. doi: 10.1111/j.1365-2613.2007.00551.x

Magnusson, S. P., and Kjaer, M. (2003). Region-specific differences in Achilles tendon cross-sectional area in runners and non-runners. Eur. J. Appl. Physiol. 90, 549-553. doi: 10.1007/s00421-003-0865-8

Malina, R. M., Rogol, A. D., Cumming, S. P., Coelho e Silva, M. J., and Figueiredo, A. J. (2015). Biological maturation of youth athletes: assessment and implications. Br. J. Sports Med. 49, 852-859. doi: 10.1136/bjsports-2015-094623

Malliaras, P., Kamal, B., Nowell, A., Farley, T., Dhamu, H., Simpson, V., et al. (2013). Patellar tendon adaptation in relation to load-intensity and contraction type. J. Biomech. 46, 1893-1899. doi: 10.1016/j.jbiomech.2013.04.022

Matos, N., and Winsley, R. J. (2007). Trainability of young athletes and overtraining. J. Sports Sci. Med. 6, 353-367.

Mersmann, F., Bohm, S., Schroll, A., Boeth, H., Duda, G., and Arampatzis, A. (2014). Evidence of imbalanced adaptation between muscle and tendon in adolescent athletes. Scand. J. Med. Sci. Spor. 24, E283-E289. doi: $10.1111 / \mathrm{sms} .12166$

Mersmann, F., Bohm, S., Schroll, A., Boeth, H., Duda, G., and Arampatzis, A. (2015). Muscle shape consistency and muscle volume prediction of thigh muscles. Scand. J. Med. Sci. Spor. 25, e208-e213. doi: 10.1111/sms.12285

Mersmann, F., Bohm, S., Schroll, A., Boeth, H., Duda, G. N., and Arampatzis, A. (2017). Muscle and tendon adaptation in adolescent athletes: a longitudinal study. Scand. J. Med. Sci. Spor. 27, 75-82. doi: 10.1111/sms.12631

Mersmann, F., Bohm, S., Schroll, A., Marzilger, R., and Arampatzis, A. (2016). Athletic training affects the uniformity of muscle and tendon adaptation during adolescence. J. Appl. Physiol. 121, 893-899. doi: 10.1152/japplphysiol.00493.2016

Moran, J., Sandercock, G. R. H., Ramírez-Campillo, R., Meylan, C., Collison, J., and Parry, D. A. (2016). A meta-analysis of maturation-related variation in adolescent boy athletes' adaptations to short-term resistance training. J. Sports Sci. 35, 1-11. doi: 10.1080/02640414.2016.1209306

Neugebauer, J. M., and Hawkins, D. A. (2012). Identifying factors related to Achilles tendon stress, strain, and stiffness before and after 6 months of growth in youth 10-14 years of age. J. Biomech. 45, 2457-2461. doi: 10.1016/j.jbiomech.2012.06.027

Ozmun, J. C., Mikesky, A. E., and Surburg, P. R. (1994). Neuromuscular adaptations following prepubescent strength training. Med. Sci. Sport Exer. 26:510.

Ramsay, J. A., Blimkie, C. J., Smith, K., Garner, S., MacDougall, J. D., and Sale, D. G. (1990). Strength training effects in prepubescent boys. Med. Sci. Sport Exer. $22,605-614$.

Roberts, T. J. (1997). Muscular force in running turkeys: the economy of minimizing work. Science 275, 1113-1115. doi: 10.1126/science.275.5303.1113 
Rosager, S., Aagaard, P., Dyhre-Poulsen, P., Neergaard, K., Kjaer, M., and Magnusson, S. P. (2002). Load-displacement properties of the human triceps surae aponeurosis and tendon in runners and non-runners. Scand. J. Med. Sci. Spor. 12, 90-98. doi: 10.1034/j.1600-0838.2002.120205.x

Sáez-Sáez de Villarreal, E., Requena, B., and Newton, R. U. (2010). Does plyometric training improve strength performance? A meta-analysis. J. Sci. Med. Sport 13, 513-522. doi: 10.1016/j.jsams.2009.08.005

Sawyer, S. M., Afifi, R. A., Bearinger, L. H., Blakemore, S.-J., Dick, B., Ezeh, A. C., et al. (2012). Adolescence: a foundation for future health. Lancet 379, 1630-1640. doi: 10.1016/S0140-6736(12)60072-5

Schulze, F., Mersmann, F., Bohm, S., and Arampatzis, A. (2012). A wide number of trials is required to achieve acceptable reliability for measurement patellar tendon elongation in vivo. Gait Posture 35, 334-338. doi: 10.1016/j.gaitpost.2011.09.107

Seynnes, O. R., de Boer, M., and Narici, M. V. (2007). Early skeletal muscle hypertrophy and architectural changes in response to high-intensity resistance training. J. Appl. Physiol. 102, 368-373. doi: 10.1152/japplphysiol.00789.2006

Shepherd, J. H., and Screen, H. R. C. (2013). Fatigue loading of tendon. Int. J. Exp. Pathol. 94, 260-270. doi: 10.1111/iep.12037

Simpson, M., Rio, E., and Cook, J. (2016). At what age do children and adolescents develop lower limb tendon pathology or tendinopathy? a systematic review and meta-analysis. Sports Med. 46, 545-557. doi: 10.1007/s40279-015-0438-0

Stafilidis, S., and Arampatzis, A. (2007). Muscle - tendon unit mechanical and morphological properties and sprint performance. J. Sports Sci. 25, 1035-1046. doi: 10.1080/02640410600951589

Stracciolini, A., Casciano, R., Levey Friedman, H., Stein, C. J., Meehan, W. P., and Micheli, L. J. (2014). Pediatric sports injuries: a comparison of males versus females. Am. J. Sports Med. 42, 965-972. doi: 10.1177/0363546514522393

Veres, S. P., Harrison, J. M., and Lee, J. M. (2013). Repeated subrupture overload causes progression of nanoscaled discrete plasticity damage in tendon collagen fibrils. J. Orthop. Res. 31, 731-737. doi: 10.1002/jor.22292

Vingren, J. L., Kraemer, W. J., Ratamess, N. A., Anderson, J. M., Volek, J. S., and Maresh, C. M. (2010). Testosterone physiology in resistance exercise and training: the up-stream regulatory elements. Sports Med. 40, 1037-1053. doi: 10.2165/11536910-000000000-00000

Waugh, C. M., Blazevich, A. J., Fath, F., and Korff, T. (2011). Age-related changes in mechanical properties of the Achilles tendon. J. Anat. 220, 144-155. doi: $10.1111 / \mathrm{j} .1469-7580.2011 .01461 . \mathrm{x}$
Waugh, C. M., Korff, T., Fath, F., and Blazevich, A. J. (2013). Rapid force production in children and adults. Med. Sci. Sport Exer. 45, 762-771. doi: 10.1249/MSS.0b013e31827a67ba

Waugh, C. M., Korff, T., Fath, F., and Blazevich, A. J. (2014). Effects of resistance training on tendon mechanical properties and rapid force production in prepubertal children. J. Appl. Physiol. 117, 257-266. doi: 10.1152/japplphysiol.00325.2014

Wiesinger, H.-P., Kösters, A., Müller, E., and Seynnes, O. R. (2015). Effects of increased loading on in vivo tendon properties. Med. Sci. Sport Exer. 47, 1885-1895. doi: 10.1249/MSS.00000000000 00603

Woo, S. L. (1982). Mechanical properties of tendons and ligaments. I. Quasi-static and nonlinear viscoelastic properties. Biorheology 19, 385-396.

World health organization: department of child and adolescent health and development (2001). The Second Decade: Improving Adolescent Health and Development. Geneva: World Health Organization.

Wren, T. A. L., Lindsey, D. P., Beaupré, G. S., and Carter, D. R. (2003). Effects of creep and cyclic loading on the mechanical properties and failure of human Achilles tendons. Ann. Biomed. Eng. 31, 710-717. doi: 10.1114/1.15 69267

Conflict of Interest Statement: The authors declare that the research was conducted in the absence of any commercial or financial relationships that could be construed as a potential conflict of interest.

The reviewer CC and handling Editor declared their shared affiliation, and the handling Editor states that the process nevertheless met the standards of a fair and objective review.

Copyright (C) 2017 Mersmann, Charcharis, Bohm and Arampatzis. This is an openaccess article distributed under the terms of the Creative Commons Attribution License (CC BY). The use, distribution or reproduction in other forums is permitted, provided the original author(s) or licensor are credited and that the original publication in this journal is cited, in accordance with accepted academic practice. No use, distribution or reproduction is permitted which does not comply with these terms. 